\title{
A 3D SEDIMENT TRANSPORT MODEL FOR COMBINED WAVE-CURRENT FLOWS
}

\author{
Peifeng $\mathrm{Ma}^{1}$ and Ole Secher Madsen ${ }^{2}$
}

\begin{abstract}
Accurate prediction of current velocity and bottom shear stress, which both can be significantly influenced by wind waves, is essential for sediment transport predictions in the coastal environment. Consequently wind-wave effects must be taken into account in a numerical sediment transport model for application in coastal waters. In the present study, elements of a large-scale 3D numerical coastal circulation and sediment transport model are developed to predict net, i.e. the wave-period-averaged, sediment transport rates. The sediment transport components considered are (i) bed-load transport; (ii) mean suspended load sediment transport within the wave boundary layer, which is obtained from an analytical solution; and (iii) suspended load sediment transport above the wave bottom boundary layer, which is obtained from a numerical model. In all model components wind wave effects are accounted for through simple analytical models. Thus, the roughness prescribed for the hydrodynamic part of the numerical coastal circulation model is the apparent roughness, i.e. the roughness experienced by a slowly varying current in the presence of waves. Similarly, the reference concentration specified for the sediment transport part of the numerical model is obtained from analytical solutions for suspended sediment concentrations within the combined wave-current bottom boundary layer. Stratification effects caused by suspended sediment are included in the large-scale numerical sediment transport model. Results of idealized tests suggest that wind wave effects can be pronounced, e.g. in some typical coastal scenarios sediment can only be mobilized when wind waves are present and accounted for. It is also shown that stratification can significantly affect suspended sediment transport rates of fine sediments.
\end{abstract}

Keywords: sediment transport; bottom boundary layer; wave-current interaction; sediment-induced stratification

\section{INTRODUCTION}

As illustrated in Figure 1, a typical coastal environment consists of wind waves, slowly varying currents, such as tide- and wind-driven flows, and sediment in the bottom and water column. Sediment can be moved as bed-load transport on the seabed or stirred up into the water column by bed shear stress and then transported by a current as suspended load transport. It is well-known that wind waves can intensify the near bottom turbulence significantly due to the limited thickness of the wave boundary layer. As a result, the bottom shear stress and turbulent mixing in the water column can be markedly enhanced by the presence of wind waves resulting in more sediment being mobilized and moved to upper layers of the water column where it is then made available for transport by currents. Consequently, wind wave effects must be included in numerical coastal circulation and sediment transport models. Since coastal circulation models cannot resolve the short time-scales governing the wave bottom boundary layer, the hydrodynamic as well as sediment transport processes within the combined wave-current bottom boundary layer must be calculated separately to produce physically realistic, slowly varying boundary conditions that are passed on to the numerical coastal circulation and sediment transport model.

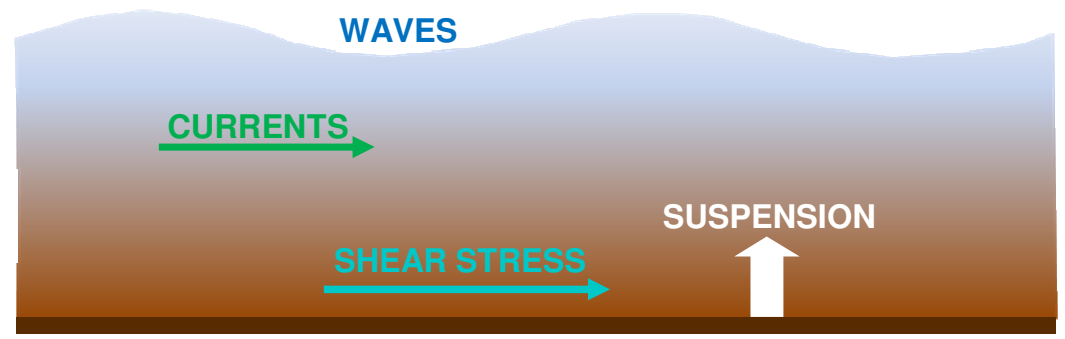

Figure 1 Illustration of a typical coastal environment

Many numerical sediment transport studies have been conducted in the past few decades (e.g. Li and Amos, 2001; Lesser et al., 2004; Warner et al., 2008). Wind wave effects have been taken into

\footnotetext{
${ }^{1}$ Singapore-MIT Alliance for Research and Technology, 1 CREATE way, \#09-03 CREATE Tower, Singapore, 138602

2 Parsons Laboratory, Massachusetts Institute of Technology, Room 48-216C, 15 Vassar Street, Cambridge, MA, 02139, USA
} 
account in some models, e.g. some wave-associated coefficients are introduced by Lesser et al. (2004) to account for wave effects on sediment transport. Wave-current interaction models (e.g. Madsen, 1994) are introduced by Li and Amos (2001) and Warner et al. (2008) to predict combined wave-current shear stresses for sediment transport predictions. However, most numerical sediment transport models treat the wave boundary layer as a portion of numerical domain, regardless of the much different physics within the layer which usually cannot be resolved by coastal circulation models. Density changes caused by suspended sediment have been included in some sediment transport models (e.g. Lesser et al., 2004; Warner et al., 2008), but hardly any information has been reported on the importance of this sediment stratification effect.

The objective of the present study is to develop a three dimensional numerical sediment transport model for combined wave-current flows. A bottom boundary layer model is introduced to account for wind wave effects on current flow and sediment transport. The mean suspended sediment transport within the boundary layer is calculated separately and analytically. The sediment stratification effect is studied. The paper is organized as follows. A model description, including flow model, bottom boundary layer model and sediment transport model, is presented in Section 2. In Section 3, numerical experiments are conducted to test the model. Finally conclusions are provided in Section 4.

\section{MODEL DESCRIPTION}

In coastal waters, sediment is usually stirred up by the bed shear stress and then transported by a current. Bed shear stress determines the amount of sediment being mobilized and flow velocity determines how far the sediment can be transported. In the present model, a bottom boundary layer model is introduced to predict bed shear stress in combined wave-current flows, including the total and sediment transport shear stresses. A numerical coastal circulation model is used to compute eddy viscosity and velocity profiles for the slowly varying currents above the bottom boundary layer, for which wave effects are incorporated through the specification of an enhanced apparent bottom roughness, i.e. the roughness experienced by a current in the presence of wind waves. The bed-load transport rate and the reference concentration for suspended sediment concentration are computed from the so-called skin friction or sediment transport shear stress. The mean suspended load transport within the bottom boundary layer is solved analytically. The suspended load sediment transport rate above the bottom boundary layer is computed by a numerical sediment transport model that solves the unsteady, i.e. slowly varying, advection-diffusion equation to obtain the suspended sediment concentration corresponding to a specified reference concentration predicted by the analytical bottom boundary layer model that includes the effects of wind waves.

The present model is formulated in a terrain following coordinate system $(x, y, \sigma)$ with $x$ and $y$ indicating the two directions in the horizontal plane and $\sigma$ representing the scaled vertical direction. The velocities in the three directions are denoted by $(u, v, w)$. The still water depth is $h$ and surface elevation is denoted by $\eta$. The water density and molecular viscosity are taken to be $\rho=1,025 \mathrm{~kg} / \mathrm{m}^{3}$ and $v=$ $1.3 \times 10^{-6} \mathrm{~m}^{2} / \mathrm{s}$, respectively. To be realistic, we assume the presence of random wind waves which have a root mean square (rms) wave height $H_{r}$ and a representative wave period $T_{r}$ or angular frequency $\omega_{r}=$ $2 \pi / T_{r}$ and assumed described by linear wave theory. Non-cohesive sediment with median diameter $d$ and density $\rho_{s}=2,650 \mathrm{~kg} / \mathrm{m}^{3}$ is considered in the present study.

\subsection{Hydrodynamic Model}

The Princeton Ocean Model (POM), Blumberg and Mellor (1987), is used to predict 3D flow velocities $(u, v, w)$ in the $x, y$ and $\sigma$ directions, respectively. POM is a primitive equation ocean model based on hydrostatic and Bousinesq assumptions, in which the turbulence closure scheme of Mellor and Yamada (1982), hereafter referred to as MY, is incorporated to estimate eddy viscosity $K_{M}$ and eddy diffusivity for heat, $K_{H}$. In the present study, we assume the suspended sediment diffuses in the same way as the heat and therefore take the sediment eddy diffusivity $K_{S}=K_{H}$. As pointed out by Warner et al. (2005), the MY scheme predicts a substantially smaller eddy viscosity than analytical solutions and other turbulence closure schemes. This under-prediction may have significant influence on suspended sediment concentrations causing it to decay too rapidly with distance from the bed. To avoid the underprediction, the wall proximity function with open channel correction proposed by Blumberg et al. (1992) is used to replace the original wall function in the MY scheme. Since POM cannot resolve the small scale wave motion, only net or wave-period averaged quantities are computed in the present study, e.g. the net bed-load transport rate and mean concentrations. 


\subsection{Bottom Boundary Layer Model}

A wave-current interaction model (Grant and Madsen, 1979; Madsen, 1994; Humbyrd and Madsen, 2010) is incorporated into POM to account for wind wave effects. The model is based on a bilinear time-invariant eddy viscosity model that is proportional to the combined maximum wavecurrent shear velocity $u_{*_{m}}$ within and to the enhanced current shear velocity $u_{*_{c}}$ above the bottom boundary layer, i.e.

$$
K_{M}(z)= \begin{cases}\kappa u_{*_{m}} z, & z \leq \delta_{c w} \\ \kappa u_{*_{c}} z, & z \geq \delta_{c w}\end{cases}
$$

The model is able to predict the physical, i.e. movable, bottom roughness $k_{n}$, the apparent bottom roughness, i.e. the enhanced bottom roughness experienced by a current in the presence of waves, $k_{n a}$, the wave boundary layer thickness $\delta_{c w}$, the total current and wave shear velocities, $u_{*_{c}}$ and $u_{*_{w m}}$, and the current and wave skin friction, or sediment transport, shear velocities $u_{*_{c s}}$ and $u_{*_{w m s}}$.

The information required to implement the wave-current interaction model include: water depth $h$; rms wave height $H_{r}$ and period $T_{r}$ or angular frequency $\omega_{r}$; current bottom shear velocity $u_{*_{c}}$ or a reference current velocity $u_{r}$ at $z=z_{r}$; and sediment diameter $d$. The solution procedure for the wavecurrent interaction can be divided into three steps.

\subsubsection{Prediction of physical bottom roughness $\boldsymbol{k}_{\boldsymbol{n}}$ based on wave and sediment specification}

It is assumed that wind waves dominate fluid-sediment interaction and therefore determine the bed condition, i.e. flat without or with sediment motion or rippled, so that the physical roughness can be predicted solely from wave information. To do this, the maximum wave bottom orbital velocity $U_{b m}$ or the excursion amplitude $A_{b m}=U_{b m} / \omega_{r}$ is computed from rms wave height, period and water depth. Then the skin friction Shields Parameter $\psi_{d}^{\prime}$ is obtained from

$$
\psi_{d}^{\prime}=\tau_{w m}^{\prime} /[\rho(s-1) g d]=0.5 f_{w}^{\prime} U_{b m}^{2} /[(s-1) g d]
$$

where $g=9.81 \mathrm{~m}^{2} / \mathrm{s}$ is the acceleration due to gravity and $s=\rho_{s} / \rho=2.59$ is the relative density of sediment. The wave skin friction factor $f_{w}^{\prime}$ is computed based on a bottom roughness equal to the median sediment diameter, i.e.

$$
f_{w}^{\prime}= \begin{cases}\exp \left[7.02 X^{-0.078}-8.82\right], & X=A_{b m} / d<10^{2} \\ \exp \left[5.61 X^{-0.109}-7.30\right], & X=A_{b m} / d \geq 10^{2}\end{cases}
$$

There will be no sediment motion if the skin friction Shields Parameter $\psi_{d}^{\prime}$ given by (2) is smaller than the critical value, $\psi_{c r}$, which can be estimated by the formula proposed by Herrmann and Madsen (2007)

$$
\psi_{c r}=0.095 S_{*}^{-2 / 3}+0.056\left[1-\exp \left(-S_{*}^{3 / 4} / 20\right)\right]
$$

where $S_{*}=d \sqrt{(s-1) g d} / 4 v$. The bed will be flat with no transport if $\psi_{d}^{\prime}<\psi_{c r}$, rippled if $\psi_{c r} \leq \psi_{d}^{\prime} \leq 0.35$ and in sheet flow condition if $\psi_{d}^{\prime}>0.35$. The threshold Shields Parameter for sheet flow condition is taken to be 0.35 , rather than the usual value of 0.7 , because the Shields Parameter computed using the significant wave orbital velocity, $\psi_{s}^{\prime}=2 \psi_{d}^{\prime}$, has been found to describe onset of sheet flow for random waves. The threshold Shields Parameter for initiation of motion is still taken as $\psi_{c r}$ in order to be consistent with the critical value used in the calculation of bed-load transport rate and reference concentration for sediment suspension in combined wave-current flows.

The physical roughness for different bed conditions can therefore be computed from

$$
k_{n}=\left\{\begin{array}{cl}
d, & \psi_{d}^{\prime}<\psi_{c r}(\text { no transport) } \\
k_{n}\left(d, A_{b m} / k_{n}\right), & \psi_{c r} \leq \psi_{d}^{\prime} \leq 0.35 \text { (rippled bed) } \\
\max \left(d, A_{n} d\right), & \psi_{d}^{\prime}>0.35 \text { (sheet flow) }
\end{array}\right.
$$


where $A_{n}$ is a function of $\psi_{d}^{\prime}$ suggested to be $15 \psi_{d}^{\prime}$ by Madsen (2002) based on very limited data.

For rippled bed conditions, the physical roughness in (5) can be calculated iteratively through the empirical relationship for the energy dissipation factor established by Humbyrd and Madsen (2010)

$$
f_{e}=0.14 S_{*}^{0.25} \exp \left(-4.94 \psi_{d}^{\prime}\right)
$$

This energy dissipation factor is a function of bottom roughness

$$
f_{e}=f_{w} \cos \left\{(\pi / 60)\left[11-2 \log _{10}\left(A_{b m} / k_{n}\right)\right]\right\}
$$

where $f_{w}$ is computed from (3) with $X=A_{b m} / k_{n}$. Therefore, the energy dissipation factor $f_{e}$ can be calculated by (6a) based on the skin friction Shields Parameter obtained from (2). Having $f_{e}$, the physical roughness can be computed iteratively from (6b).

\subsubsection{Prediction of total shear velocities, boundary layer thickness and apparent roughness}

With the physical bottom roughness $k_{n}$ obtained as outlined in Section 2.2.1, and the current shear velocity $u_{*_{c}}$, a wave-current interaction analysis can be performed to yield the maximum wave shear velocity, $u_{*_{w m}}$. The general procedure is

$$
\begin{aligned}
C_{\mu} & =\left(1+2\left|\cos \phi_{c w}\right| \mu+\mu^{2}\right)^{1 / 2} \text { with } \mu=u_{*_{c}}^{2} / u_{*_{w m}}^{2} \\
f_{c w} & =\left\{\begin{array}{l}
C_{\mu} \exp \left[7.02 X_{\mu}^{-0.078}-8.82\right], \quad X_{\mu}=C_{\mu} A_{b m} / k_{n}<10^{2} \\
C_{\mu} \exp \left[5.61 X_{\mu}^{-0.109}-7.30\right], \quad X_{\mu}=C_{\mu} A_{b m} / k_{n} \geq 10^{2}
\end{array}\right. \\
u_{*_{w m}} & =\sqrt{f_{c w} / 2} U_{b m}
\end{aligned}
$$

where $\phi_{c w}=\phi_{c}-\phi_{w}$ is the angle between currents and waves, with $\phi_{c}$ and $\phi_{w}$ denoting the current and wave directions with respect to the $x$ axis. In this wave-current interaction procedure, the current shear stress or shear velocity $u_{*_{c}}$ is kept unchanged since it would be slowly varying. But this does not mean the current shear stress is fixed for the entire simulation, since it varies slowly with time as predicted by the numerical circulation model and/or slowly changing wind wave conditions. Hence, the bottom boundary layer model and the flow model are fully coupled.

Once the iteration procedure in (7a-c) converges, we can obtain $u_{*_{c}}$ and $u_{w_{w m}}$ and the combined maximum shear velocity $u_{*_{m}}=\sqrt{C_{\mu}} u_{*_{w m}}$, from which we can calculate the bottom boundary layer thickness

$$
\delta_{c w}=P A_{\mu} \kappa u_{*_{m}} / \omega_{r}
$$

where

$$
\begin{aligned}
& A_{\mu}=\exp \left[2.96\left(C_{\mu} A_{b m} / k_{n}\right)^{-0.071}-1.45\right] \\
& P=\frac{1}{16.3}\left(\sqrt{C_{\mu} / \mu}\right)^{\sqrt{C_{\mu}} /\left(\sqrt{C_{\mu}}-\sqrt{\mu}\right)}
\end{aligned}
$$

The current velocity within and above the boundary layer can now be computed from

$$
u_{c}(z)= \begin{cases}u_{*_{c}}^{2} / \kappa u_{*_{m}} \cdot \ln \left(30 z / k_{n}\right), & z \leq \delta_{c w} \\ u_{*_{c}} / \kappa \cdot \ln \left(30 z / k_{n a}\right), & z \geq \delta_{c w}\end{cases}
$$

where the apparent roughness, $k_{n a}$, is obtained by matching the velocity at $z=\delta_{c w}$

$$
k_{n a}=30 \delta_{c w}\left(30 \delta_{c w} / k_{n}\right)^{-u_{c} c u_{m}}
$$

The apparent roughness is the roughness experienced by the current in the presence of wind waves, which is used to compute the quadratic bottom friction factor 


$$
C_{D}=\kappa^{2} /\left[\ln \left(30 z_{1} / k_{n a}\right)\right]^{2}
$$

where $z_{1}$ is the first interior velocity grid level above the bottom in POM simulations . A logarithmic velocity distribution near the bottom is assumed to obtain (11). Through this bottom friction factor, $C_{D}$, the numerical circulation model captures the enhancement of bottom resistance induced by wind waves. The apparent roughness is the only parameter passing from the bottom boundary layer model to the flow model, which in turn will provide an updated current shear stress to the bottom boundary layer model. The current bottom shear stress $\tau_{b c}$ in POM is computed from the friction factor and the horizontal velocity $U_{l}$ at the level $z=z_{1}$

$$
\tau_{b c}=\rho C_{D} U_{1}^{2}
$$

\subsubsection{Prediction of sediment transport shear stress}

To compute sediment transport rates, we need to evaluate the shear stresses or shear velocities responsible for moving and entraining sediment. For sheet flow conditions, the shear stresses obtained as outlined above are the sediment transport shear stresses. For rippled bed conditions, the total shear stresses obtained in the preceding section consist of skin friction and form drag, and of these only the skin friction shear stress is responsible for sediment motion. Thus, when the bed is rippled, the skin friction shear stress needs to be separated from the total shear stress. To do this, another wave-current interaction analysis is performed based on a reference current velocity and a skin friction roughness.

For rippled bed conditions, a reasonable skin friction roughness should be related to the flow intensity rather than a constant multiple of the grain size. In order to make a smooth transition from rippled bed to sheet flow conditions, we adopt the roughness for sheet flow condition in (5) to calculate skin friction bottom roughness, i.e. we take

$$
k_{n s}=\max \left(d, 15 \psi_{d}^{\prime} d\right)
$$

Unfortunately, no experimental data are available to support a specific choice of the reference level for the reference current velocity to be used in the computation of the sediment transport shear stresses for combined wave-current flows. Thus we choose, somewhat arbitrarily but physically and computationally pleasing, the upper boundary of the wave bottom boundary layer as the reference level at which the reference current velocity is specified, i.e. we have from (9)

$$
u_{r}=\left(u_{*_{c}} / \kappa\right) \ln 30 \delta_{c w} / k_{n a} \text { at } z_{r}=\delta_{c w}
$$

Hence the sediment transport shear velocities $u_{*_{c s}}$ and $u_{*_{w m s}}$ can be computed from the following iterative procedure

$$
\begin{aligned}
& C_{\mu s}=\left(1+2\left|\cos \phi_{c w}\right| \mu_{s}+\mu_{s}^{2}\right)^{1 / 2} \text { with } \mu_{s}=u_{* c s}^{2} / u_{*_{w m s}}^{2} \\
& f_{c w s}=\left\{\begin{array}{l}
C_{\mu s} \exp \left[7.02 X_{s}^{-0.078}-8.82\right], \quad X_{s}=C_{\mu s} A_{b m} / k_{n s}<10^{2} \\
C_{\mu s} \exp \left[5.61 X_{s}^{-0.109}-7.30\right], X_{s}=C_{\mu s} A_{b m} / k_{n s} \geq 10^{2}
\end{array}\right. \\
& u_{*_{w m s}}^{2}=0.5 f_{c w s} U_{b m}^{2} \\
& u_{*_{m s}}=\sqrt{C_{\mu s}} u_{*_{w m s}} \\
& u_{*_{c s}}=u_{*_{m s}} \frac{\ln \left(z_{r} / \delta_{c w s}\right)}{\ln \left(\delta_{c w s} / z_{0 s}\right)}\left[-\frac{1}{2}+\sqrt{\left.\frac{1}{4}+\frac{\kappa u_{r} \ln \left(\delta_{c w s} / z_{0 s}\right)}{u_{* m s} \ln \left(z_{r} / \delta_{c w s}\right)^{2}}\right]}\right.
\end{aligned}
$$

where $z_{0 s}=k_{n s} / 30$ and the thickness $\delta_{c w s}$ is computed in the same way as $\delta_{c w}$ in (8a-c) but with the skin friction parameters $k_{n s}, \mu_{s}, C_{\mu s}$ and $u_{*_{m s}}$. After the iteration procedure in (15a-e) converges, we can obtain the sediment transport shear stresses for the current, $\tau_{c s}=\rho u_{*_{c s}}^{2}$, and for the waves, $\tau_{w m s}=\rho u_{*_{w m s}}^{2}$. The total sediment transport shear stress can then be written as 


$$
\begin{aligned}
\vec{\tau}_{b s}(t) & =\left[\begin{array}{ll}
\tau_{b s x}(t), & \tau_{b s y}(t)
\end{array}\right] \\
& =\left[\tau_{w m s} \cos \omega_{r} t \cos \phi_{w}+\tau_{c s} \cos \phi_{c}, \tau_{w m s} \cos \omega_{r} t \sin \phi_{w}+\tau_{c s} \sin \phi_{c}\right]
\end{aligned}
$$

\subsection{Sediment Transport Model}

Instantaneous sediment transport rates are averaged over a wind wave period to yield mean or net sediment transport rates. Three categories of sediment transport are considered: (i) Net bed load transport, $\overline{\boldsymbol{q}}_{\boldsymbol{B}}$, which takes place below the reference level for mean concentration specification for the suspended load, $z=7 d$; (ii) Mean suspended load transport within the wave boundary layer, $7 d<z<$ $\delta_{c w}, \overline{\boldsymbol{q}}_{s 1}$, which is obtained from analytical solutions for current velocity and mean sediment concentration within the wave boundary layer, since POM does not resolve wave boundary layer scales; and (iii) Mean suspended load transport above the wave boundary layer, $z>\delta_{c w}, \overline{\boldsymbol{q}}_{s 2}$, which is obtained by solving an advection-diffusion equation for the mean sediment concentration and the current velocity using POM. (1991).

The instantaneous bed-load mass transport rate is computed by the model proposed by Madsen

$$
\vec{q}_{B}(t)=\operatorname{Max}\left\{\left|\vec{\tau}_{b s}(t)-\tau_{c r, \beta}\right|, 0\right\} \cdot \frac{8 \rho_{s}}{\rho^{1.5}(s-1) g} \cdot \frac{\left(\sqrt{\left|\vec{\tau}_{b s}\right|}-\alpha_{\beta} \sqrt{\tau_{c r, \beta}}\right)}{\tan \varphi_{m}-\tan \beta} \cdot \frac{\vec{\tau}_{b s}}{\left|\vec{\tau}_{b s}\right|}
$$

where $\beta$ is the bottom slope in the direction of the instantaneous sediment transport shear stress and considered positive when sloping up in the shear stress direction. In large scale coastal simulations, the bed slope is usually very small, i.e. $\tan \beta \approx \sin \beta \approx \beta<<1$. The critical shear stress with slope effect is given by $\tau_{c r, \beta}=\tau_{c r}\left(1+\tan \beta / \tan \varphi_{s}\right)$ with $\tau_{c r}=\psi_{c r}[\rho(s-1) g d]$ and $\psi_{c r}$ computed by (4). $\varphi_{s}$ and $\varphi_{m}$ are static and dynamic friction angles for sediment which have values of $30^{\circ}$ and $50^{\circ}$, respectively. Thus, the parameter $\alpha_{\beta}=\sqrt{\left(\tan \varphi_{m}+\tan \beta\right) /\left(\tan \varphi_{s}+\tan \beta\right)} \approx 0.7$.

The net bed-load transport rate can be computed by averaging (17) over a wave period as

$$
\overline{\boldsymbol{q}}_{\boldsymbol{B}}=\frac{1}{T} \int_{0}^{T} \vec{q}_{B}(t) d t
$$

The instantaneous bed-load transport rate for a pure sinusoidal wave condition is $\vec{q}_{B}(t) \propto \sqrt{\left|\tau_{w m s} \cos \omega_{r} t\right|} \cdot \tau_{w m s} \cos \omega_{r} t$, which obviously leads to a period-averaged bed-load transport of zero. Thus, wind waves can only give rise to a non-zero net bed-load transport when they are acting together with a current and/or on a sloping bottom. For example, if we assume wave shear stress to be dominant and the bed slope to be small, the net bed-load transport was shown by Madsen (2002) to be approximately given by $\overline{\boldsymbol{q}}_{\boldsymbol{B}} \propto 9 u_{*_{w m s}}\left(u_{*_{c s}}^{2}-u_{*_{w m s}}^{2} \tan \beta / 2 \tan \varphi_{m}\right)$.

To predict suspended load transport rate above the bed-load transport layer, i.e. $z>7 d$, a reference concentration at $z=7 d$, related to the excess sediment transport shear stress, was introduced by Madsen (2002)

$$
C_{R}(t)=0.0022 \rho_{s} c_{0} \max \left[\left(\left|\vec{\tau}_{b s}(t)\right| / \tau_{c r, \beta}-1\right), 0\right] \text { at } z=7 d
$$

where $c_{0}=0.65$ is the volume concentration of sediment in the bed. Again, only the mean reference concentration is considered here, which is

$$
\bar{C}_{R}=\frac{1}{T} \int_{0}^{T} C_{R}(t) d t
$$

It can be shown that, unlike the net bed-load transport, linear wind waves alone can produce a significant mean reference concentration, e.g. the mean concentration for pure wave conditions can be expressed by $\bar{C}_{R}=0.0044 / \pi \rho_{s} c_{0}\left(\tau_{w m s} / \tau_{c r}-1\right)$ which obviously does not vanish. Therefore, waveinduced bottom shear stress can play a very important role in predicting suspended load transport and 
should not be neglected, especially for wave dominated conditions which are common in most coastal waters.

Since the large scale numerical model does not resolve wave bottom boundary layer scales, the mean concentration in this layer is computed analytically based on an eddy diffusivity $K_{S}$ which is assumed identical to the eddy viscosity, $K_{M}$, given by (1). To obtain the analytical concentration solution, we assume the time scale of vertical diffusion in the boundary layer is much smaller than the scale of the slow current flow changes so that equilibrium can be assumed. This assumption should be reasonable, since the wave boundary layer is thin and the turbulent mixing is very strong. For equilibrium, the vertical diffusion and the sediment settling effects are balanced to yield an analytical concentration solution within the wave boundary layer

$$
\bar{C}(z)=\bar{C}_{R}(z / 7 d)^{-w_{f} / \kappa u_{m}}
$$

where $w_{f}$ is the sediment fall velocity. It is calculated from the formula proposed by Jiménez and Madsen (2003)

$$
w_{f}=\sqrt{(s-1) g d_{n}} /\left(0.95+5.1 / S_{*_{n}}\right)
$$

where $d_{n} \approx d / 0.9$ and $S_{*_{n}}=d_{n} \sqrt{(s-1) g d_{n}} / 4 v$.

Therefore the net suspended load transport rate in the wave bottom boundary layer is

$$
\bar{q}_{S 1}=\int_{7 d}^{\delta_{c w}} \vec{u}_{c} \bar{C} d z
$$

where the current velocity vector $\vec{u}_{c}(z)$ is given by (9). This integration of (23) can be performed analytically (e.g. Madsen, 2002) or numerically by discretizing the boundary layer into a large number of layers. In the present study, the latter method is adopted.

The hydrodynamic and sediment transport processes above the boundary layer are far more complex than their counterparts within the bottom boundary layer and are solved numerically in the present study. The slowly varying current velocity is computed from the numerical circulation model, POM, whereas the mass concentration $C(z)$ is obtained by numerically solving the advection-diffusion equation for suspended sediment,

$$
\begin{aligned}
& \frac{\partial D C}{\partial t}+\frac{\partial}{\partial x}(D u C)+\frac{\partial}{\partial y}(D v C)+\frac{\partial}{\partial \sigma}\left[\left(w-w_{f}\right) C\right]= \\
& \frac{\partial}{\partial \sigma}\left(\frac{K_{S}}{D} \frac{\partial C}{\partial \sigma}\right)+\frac{\partial}{\partial x}\left(A_{H} D \frac{\partial C}{\partial x}\right)+\frac{\partial}{\partial y}\left(A_{H} D \frac{\partial C}{\partial y}\right)
\end{aligned}
$$

where $D=h+\eta$ is the total water depth with $\eta$ the free surface elevation predicted by POM, $A_{H}$ is the horizontal eddy diffusivity which is assumed the same as the horizontal eddy viscosity and predicted by POM, and the vertical diffusivity for sediment, $K_{S}$, is assumed identical to the heat diffusivity predicted by MY turbulence closure scheme in POM.

Boundary conditions need to be specified in order to solve (24). At land boundaries, a no flux condition is applied, i.e. $\partial C / \partial n=0$ with $n$ denoting the outward normal direction at the boundary. At open boundaries, concentration is specified for inflow condition, i.e. $C=C_{i n}$ if $u_{n}<0$ with $u_{n}$ the flow velocity in the outward normal direction, $n$, and an advection condition is applied for outflow, i.e. $\partial C / \partial t+u_{n} \partial C / \partial n=0$ if $u_{n}>0$. At the free surface, zero net sediment flux is assured by setting $K_{S} \partial C / \partial \sigma+D w_{f} C=0$ at $z=h+\eta$, whereas a sediment concentration, the reference concentration, is specified as $C=C_{b}$ at bottom.

To obtain the reference concentration, $C_{b}$, we first compute the mean concentration at the outer edge of the wave boundary layer, i.e. at $z=\delta_{c w}$, from the analytical wave boundary layer solution, (21),

$$
C_{a}=\bar{C}\left(z=\delta_{c w}\right)=\bar{C}_{R} \cdot\left(\delta_{c w} / 7 d\right)^{-w_{f} / \kappa u_{m}}
$$

Physically, the bottom level in POM simulations is defined at the outer edge of the wave boundary layer, i.e. $z=\delta_{c w}$. However, due to the staggered grid system used in POM, the bottom level for 
concentration is not at $z=\delta_{c w}$ but a half grid spacing above it, i.e. at $z=z_{b}=\delta_{c w}+\Delta z_{b} / 2$ with $\Delta z_{b}$ the spacing for the interior grid cell immediately above the bottom. Assuming equilibrium conditions to apply within the bottom half of the first grid, the concentration $C_{b}$ can be obtained analytically based on the reference concentration $C_{a}$ at $z=\delta_{c w}$ and the linear eddy diffusivity above the boundary layer given by (1). In this manner, the reference concentration for the large scale numerical sediment transport model clearly accounts for wind wave effects and it becomes

$$
C_{b}=C_{a}\left(1+\Delta z_{b} / 2 \delta_{c w}\right)^{-w_{f} / \kappa u_{c}} \text { at } z=z_{b}
$$

The eddy diffusivity predicted by POM approaches zero at the bottom level which is actually at the outer edge of the wave boundary layer where the eddy diffusivity according to the wave boundary layer model should be $\kappa u_{*} \delta_{c w}$. This relatively small eddy diffusivity may produce a large difference in sediment concentration, since suspended sediment concentration is very sensitive to the diffusivity near the bottom. For example, if we assume a typical condition with $w_{f}=1 \mathrm{~cm} / \mathrm{s}, \quad u_{*_{c}}=2.5 \mathrm{~cm} / \mathrm{s}$, $\delta_{c w}=5 \mathrm{~cm}, \Delta z_{b}=10 \mathrm{~cm}$ and $C_{a}=1 \mathrm{~kg} / \mathrm{m}^{3}$, we obtain $C_{b}=0.5 \mathrm{~kg} / \mathrm{m}^{3}$ from (26), and the concentration at the upper boundary of the first grid cell, $z=\delta_{c w}+\Delta z_{b}$ is roughly $0.33 \mathrm{~kg} / \mathrm{m}^{3}$. If directly using the eddy diffusivity predicted by POM, the concentration at $z=\delta_{c w}+\Delta z_{b}$ predicted by the present model can be estimated analytically to be $0.25 \mathrm{~kg} / \mathrm{m}^{3}$. This underestimates the concentration by about $24 \%$. To avoid this under-predictions and be more physically consistent, we add the constant eddy diffusivity, $\kappa u_{*_{c}} \delta_{c w}$, to the POM predicted eddy diffusivity $K_{\text {SPOM }}$ over the entire water depth

$$
K_{S}=K_{S P O M}+\kappa u_{*_{c}} \delta_{c w}
$$

This adjustment may lead to some minor error in eddy diffusivity near the surface as it does not approach zero. However, this error should have very little influence on the predicted sediment concentration as it is usually quite small near the free surface.

Having the flow velocity and the concentration, the suspended load transport rate above the wave boundary layer is computed by

$$
\overline{\boldsymbol{q}}_{S 2}=\int_{\delta_{c w}}^{h+\eta} \vec{u} C d z
$$

Suspended sediment will change the water-sediment mixture density and therefore produce stratification, which in turn will affect the predictions of flow velocity and sediment concentration. The density of the water-sediment mixture can be computed from

$$
\rho_{\text {mix }}=\rho+C(z)(1-1 / s)
$$

A stable stratification, which would generally be the case for sediment suspended in the water column since the concentration and therefore the mixture density would decrease with distance above the bottom, suppresses the turbulence and therefore weakens the turbulent mixing. This would result in an increase in velocity and a reduction in sediment concentration. In contrast, an unstable stratification intensifies the flow turbulence and therefore results in more sediment transport. Due to the sediment settling effect, positive stratification should be more common. Unstable stratification may, however, occur in rapidly varying flows when the near-bed concentration, which responds nearly instantaneously to the reference concentration, decreases so rapidly that high sediment concentrations in upper layers have insufficient time to settle out of suspension.

\section{MODEL TESTS}

In this section, a few idealized tests are performed to examine the model's ability to predict wind wave effects and sediment transport in combined wave-current flows. The computational domain is an open channel of $100 \mathrm{~km}$ length, $10 \mathrm{~km}$ width and $10 \mathrm{~m}$ depth. In the numerical simulations, uniform horizontal grid cells of $\Delta x=\Delta y=2 \mathrm{~km}$ and 40 non-uniform sigma layers with finer spacing near the bed are used. The external and internal time steps are $6 \mathrm{sec}$ and $120 \mathrm{sec}$, respectively.

In the tests, Coriolis force is neglected. A steady open channel flow is considered which is driven by a prescribed constant volume discharge at both open boundaries, equivalent to applying a depth- 
averaged velocity $U=0.5 /(1+\eta / h) \mathrm{m} / \mathrm{s}$ at the boundaries. This leads to $U=0.5 \mathrm{~m} / \mathrm{s}$ at the center of the domain where the surface elevation $\eta=0$ after a steady state is established. The flow is uniform in the cross-channel direction. A random wind wave condition with a rms height of $H_{r}=1 \mathrm{~m}$ and a representative period of $T_{r}=8 \mathrm{sec}$ is specified. The waves are assumed to propagate in the same direction as the current flow, i.e. this is a co-directional combined wave-current flow case. Uniform sediment with a median grain size of $d=0.1 \mathrm{~mm}$ or $0.2 \mathrm{~mm}$ is assumed. This corresponds to a critical shear stress of $\tau_{c r}=0.19 \mathrm{~N} / \mathrm{m}^{2}$ or $0.20 \mathrm{~N} / \mathrm{m}^{2}$ or a critical shear velocity of $u_{* r}=1.36 \mathrm{~cm} / \mathrm{s}$ or $1.40 \mathrm{~cm} / \mathrm{s}$ as computed from (4) and a fall velocity of $w_{f}=0.72 \mathrm{~cm} / \mathrm{s}$ or $2.2 \mathrm{~cm} / \mathrm{s}$ from (22). For the inflow boundary condition, the sediment concentration is assumed zero. This creates a transition region near the upstream boundary. The length of this transition region can be estimated approximately from $L=U h^{2} / K_{S} \sim 0.5 \times 10^{2} / 0.01=5 \mathrm{~km}$. Evidently, this is a very short distance compared to the domain length of $100 \mathrm{~km}$, most of which will therefore be unaffected by transition effects.

The simulations are run for 3 days. To avoid simulation crash, the forcing is imposed gradually with a linear ramp up during the first 12 hours. The results show that steady state is established within a few hours after the ramp up period. All the results shown in this Section are obtained at the center of the domain where the surface elevation is close to zero at a time corresponding to the end of the simulation, $\mathrm{t}=72$ hours.

For the $0.1 \mathrm{~mm}$ sediment, three simulations are performed: (1) Pure current case; (2) Combined wave-current case without stratification; (3) Combined wave-current case with stratification. For the $0.2 \mathrm{~mm}$ sediment, only simulations (2) and (3) are performed. Some predicted parameters for simulations (2) and (3) are listed in Table 1, including physical bottom roughness, apparent roughness, boundary layer thickness, wave and current shear velocities and reference concentrations. Sediment transport rates are listed in Table 2 for the different simulations.

\subsection{Effects of Wind Waves}

The vertical profiles of eddy viscosity and horizontal velocity from simulations (1) and (2) for $0.1 \mathrm{~mm}$ sediment are shown in Figure 2. For the pure current simulation, the physical roughness is chosen to be the larger one of the sediment diameter $d$ and $3.3 v / u_{*_{c}}$, i.e. the simulation starts with $k_{n}=d$ by assuming a rough turbulent flow, then checks and switches to smooth turbulent flow with $k_{n}=$ $3.3 v / u_{*_{c}}$ if $d<3.3 v / u_{*_{c}}$. The flow model predicts a bottom shear velocity of $1.5 \mathrm{~cm} / \mathrm{s}$, indicating a smooth turbulent flow condition and therefore the roughness is $k_{n}=3.3 v / u_{*_{c}}=0.28 \mathrm{~mm}$. When wind waves are present, a rippled bed is obtained, leading to a much larger physical roughness of $k_{n}=1.66 \mathrm{~cm}$ and an apparent roughness of $k_{n a}=11.1 \mathrm{~cm}$ (non-stratified case in Table 1) and a bottom shear velocity of $2.72 \mathrm{~cm} / \mathrm{s}$. As a result, the maximum eddy viscosity (seen in Figure $2 \mathrm{a}$ ) almost doubles from $0.01 \mathrm{~m}^{2} / \mathrm{s}$ to $0.02 \mathrm{~m}^{2} / \mathrm{s}$ due to the presence of wind waves. The current velocity profiles in Figure $2 \mathrm{~b}$ show that the presence of wind waves leads to a lower current velocity near the bed due to the larger flow resistance. The velocity at the first interior grid point is decreased from about $0.3 \mathrm{~m} / \mathrm{s}$ for the pure current condition to $0.15 \mathrm{~m} / \mathrm{s}$ in the presence of wind waves.

\begin{tabular}{|c|c|c|c|c|}
\hline \multirow{2}{*}{$\begin{array}{c}\text { Diameter } \\
\text { Stratified } \\
\text { Parameters }\end{array}$} & \multicolumn{2}{|c|}{$0.1 \mathrm{~mm}$} & \multicolumn{2}{|c|}{$0.2 \mathrm{~mm}$} \\
\hline & No & Yes & No & Yes \\
\hline$k_{n}(\mathrm{~cm})$ & 1.66 & 1.66 & 12.3 & 12.3 \\
\hline$k_{\text {na }}(\mathrm{cm})$ & 11.1 & 14.1 & 51.8 & 53.1 \\
\hline$\delta_{c w}(\mathrm{~cm})$ & 1.82 & 1.91 & 4.31 & 4.35 \\
\hline$u_{*_{\mathrm{c}}}(\mathrm{cm} / \mathrm{s})$ & 2.72 & 2.24 & 3.43 & 3.34 \\
\hline$u^{*} \mathrm{cs}(\mathrm{cm} / \mathrm{s})$ & 1.24 & 1.00 & 0.88 & 0.85 \\
\hline$u^{*}{ }^{*} w m(c m / s)$ & 5.30 & 5.20 & 8.14 & 8.12 \\
\hline$u^{*}$ wms $(\mathrm{cm} / \mathrm{s})$ & 2.82 & 2.76 & 2.80 & 2.80 \\
\hline $\bar{C}_{R}\left(\mathrm{~kg} / \mathrm{m}^{3}\right)$ & 7.23 & 6.75 & 6.39 & 6.34 \\
\hline $\mathrm{C}_{\mathrm{a}}\left(\mathrm{kg} / \mathrm{m}^{3}\right)$ & 2.72 & 2.38 & 0.75 & 0.73 \\
\hline
\end{tabular}



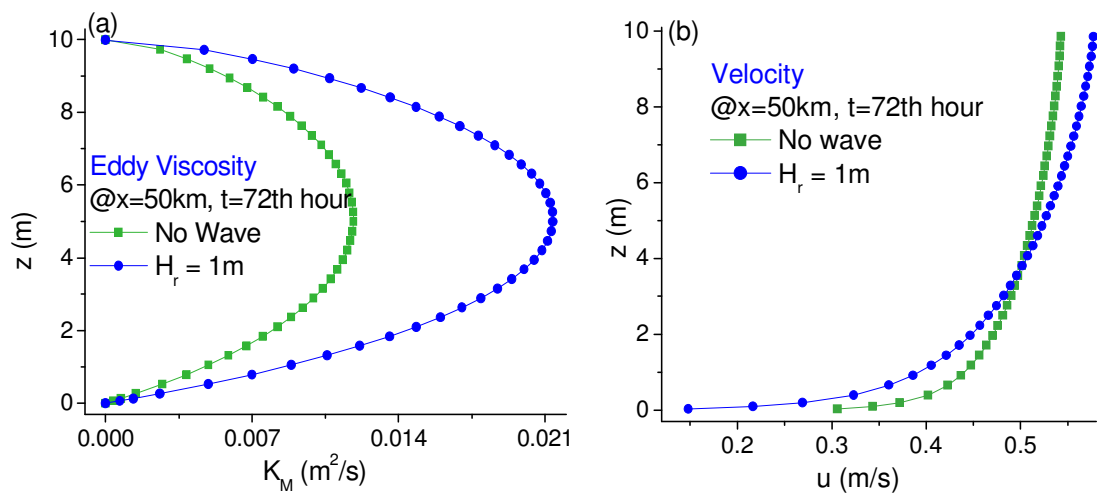

Figure 2. Predicted vertical profiles of (a) eddy viscosity and (b) current velocity for pure current and combined wave-current cases at the center of domain. Sediment size is $0.1 \mathrm{~mm}$.

Due to the flat bed condition, the sediment transport shear velocity for the pure current condition is equal to the total value of $1.5 \mathrm{~cm} / \mathrm{s}$, which is very close to the critical shear velocity of $1.36 \mathrm{~cm} / \mathrm{s}$ for the $0.1 \mathrm{~mm}$ sediment. This means that there is essentially no sediment transport for the pure current condition. In the presence of wind waves the sediment transport shear velocities are calculated to be $1.24 \mathrm{~cm} / \mathrm{s}$ and $2.82 \mathrm{~cm} / \mathrm{s}$ for current and waves, respectively, and are based on a reference current velocity of $u_{r}=11.1 \mathrm{~cm} / \mathrm{s}$ at $z_{r}=1.82 \mathrm{~cm}$ and a skin friction roughness of $k_{n s}=0.45 \mathrm{~mm}$. Hence, the sediment transport shear stress in this co-directional flow case is $\vec{\tau}_{b s}(t)=(0.8 \cos \omega t+0.15,0) \mathrm{N} / \mathrm{m}^{2}$.

This gives a maximum instantaneous value of $0.95 \mathrm{~N} / \mathrm{m}^{2}$, which is much larger than the critical shear stress of $0.19 \mathrm{~N} / \mathrm{m}^{2}$. The rather dramatic effect of the presence of wind waves is seen in the reference concentrations, $7.23 \mathrm{~kg} / \mathrm{m}^{3}$ and $2.72 \mathrm{~kg} / \mathrm{m}^{3}$ (Table 1), at the lower and upper boundaries of the wave boundary layer. As shown in Table 2, the predicted transport rates are $0.01 \mathrm{~kg} / \mathrm{m} / \mathrm{s}, 0.0051 \mathrm{~kg} / \mathrm{m} / \mathrm{s}$ and $0.5 \mathrm{~kg} / \mathrm{m} / \mathrm{s}$ for the net bed-load transport, the mean suspended load transport within and above the boundary layer, respectively. It can be seen that suspended load transport in the water column is dominant as it is about 30 times the combined bed-and suspended load transport rate within the wave boundary layer. This is mainly caused by the small fall velocity of the fine sediment and strong turbulent mixing enhanced by wind waves. Due to the thin boundary layer and fine sediment, the mean suspended load transport rate within the boundary layer is much smaller than that in the overlying water column, but it is still comparable to the net bed-load transport rate.

\begin{tabular}{|c|c|c|c|c|c|c|c|c|}
\hline & \multicolumn{2}{|c|}{$\overline{\boldsymbol{q}}_{B}$} & \multicolumn{2}{|c|}{$\overline{\boldsymbol{q}}_{s 1}$} & \multicolumn{2}{|c|}{$\overline{\boldsymbol{q}}_{s 2}$} & \multicolumn{2}{|c|}{$\overline{\boldsymbol{q}}_{T}$} \\
\hline $\begin{array}{r}\text { Stratified } \\
\text { Diameter } d\end{array}$ & No & Yes & No & Yes & No & Yes & No & Yes \\
\hline $\begin{array}{l}0.1 \mathrm{~mm} \\
0.2 \mathrm{~mm}\end{array}$ & $\begin{array}{l}1.0 \times 10^{-2} \\
62 \times 10^{-3}\end{array}$ & $\begin{array}{l}7.3 \times 10^{-3} \\
59 \times 10^{-3}\end{array}$ & $\begin{array}{l}5.1 \times 10^{-3} \\
3.1 \times 10^{-3}\end{array}$ & $\begin{array}{l}3.5 \times 10^{-3} \\
29 \times 10^{-3}\end{array}$ & $\begin{array}{l}5.0 \times 10^{-1} \\
16 \times 10^{-2}\end{array}$ & $\begin{array}{l}1.2 \times 10^{-1} \\
1.3 \times 10^{-2}\end{array}$ & $\begin{array}{l}5.2 \times 10^{-1} \\
25 \times 10^{-2}\end{array}$ & $\begin{array}{l}1.3 \times 10^{-1} \\
22 \times 10^{-2}\end{array}$ \\
\hline
\end{tabular}

\subsection{Effects of Sediment-Induced Self-stratification for $0.1 \mathrm{~mm}$ Sediments}

Sediment stratification effects are accounted for in the present study by considering the stratification effects associated with the varying density of the water-sediment mixture as expressed in (29). The routine available in POM to account for stratification due to heat and/or salinity gradient is used to account for sediment stratification effects. In general, the stratification effect is incorporated by relating a turbulent kinetic energy (TKE) production to the vertical density gradient. This leads to an enhanced turbulence intensity by a positive vertical density gradient and vice versa. In this steady flow case, the concentration decreases all the way from the bed to surface, leading to stably stratified conditions which mean that the turbulence intensity will be reduced and therefore turbulent mixing will be weakened. This is confirmed by the decreased current shear velocity of $2.24 \mathrm{~cm} / \mathrm{s}$ predicted by POM and listed in Table 1 for $0.1 \mathrm{~mm}$ sediment when stratification is accounted for compared to $2.72 \mathrm{~cm} / \mathrm{s}$ for 
the non-stratified case. As a result, the current velocity within the boundary layer (seen in Figure 3a) shows quite a significant reduction, e.g. the velocity at the outer edge of the boundary layer is decreased from $11 \mathrm{~cm} / \mathrm{s}$ to $8 \mathrm{~cm} / \mathrm{s}$. It should be recalled that stratification effects within the wave boundary layer are not accounted for, and this is the reason why the wave shear velocity, as seen in Table 1, hardly changes (it goes from $5.3 \mathrm{~cm} / \mathrm{s}$ to $5.2 \mathrm{~cm} / \mathrm{s}$ ). The reduced current shear velocity, in addition to the reduced mixing associated with stable stratification, makes the eddy diffusivity above the wave boundary layer decrease substantially as shown in Figure $4 \mathrm{a}$, reducing the maximum value from about $0.027 \mathrm{~m}^{2} / \mathrm{s}$ to $0.015 \mathrm{~m}^{2} / \mathrm{s}$.
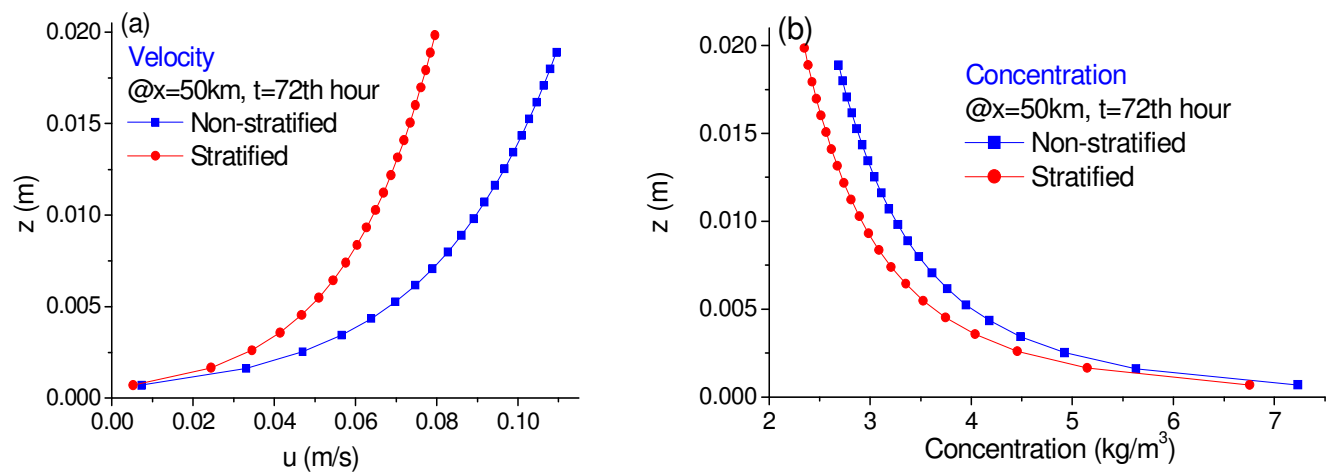

Figure 3 Analytical solutions of vertical profiles of (a) current velocity and (b) sediment concentration within the wave boundary layer at the center of domain. Sediment size is $0.1 \mathrm{~mm}$.
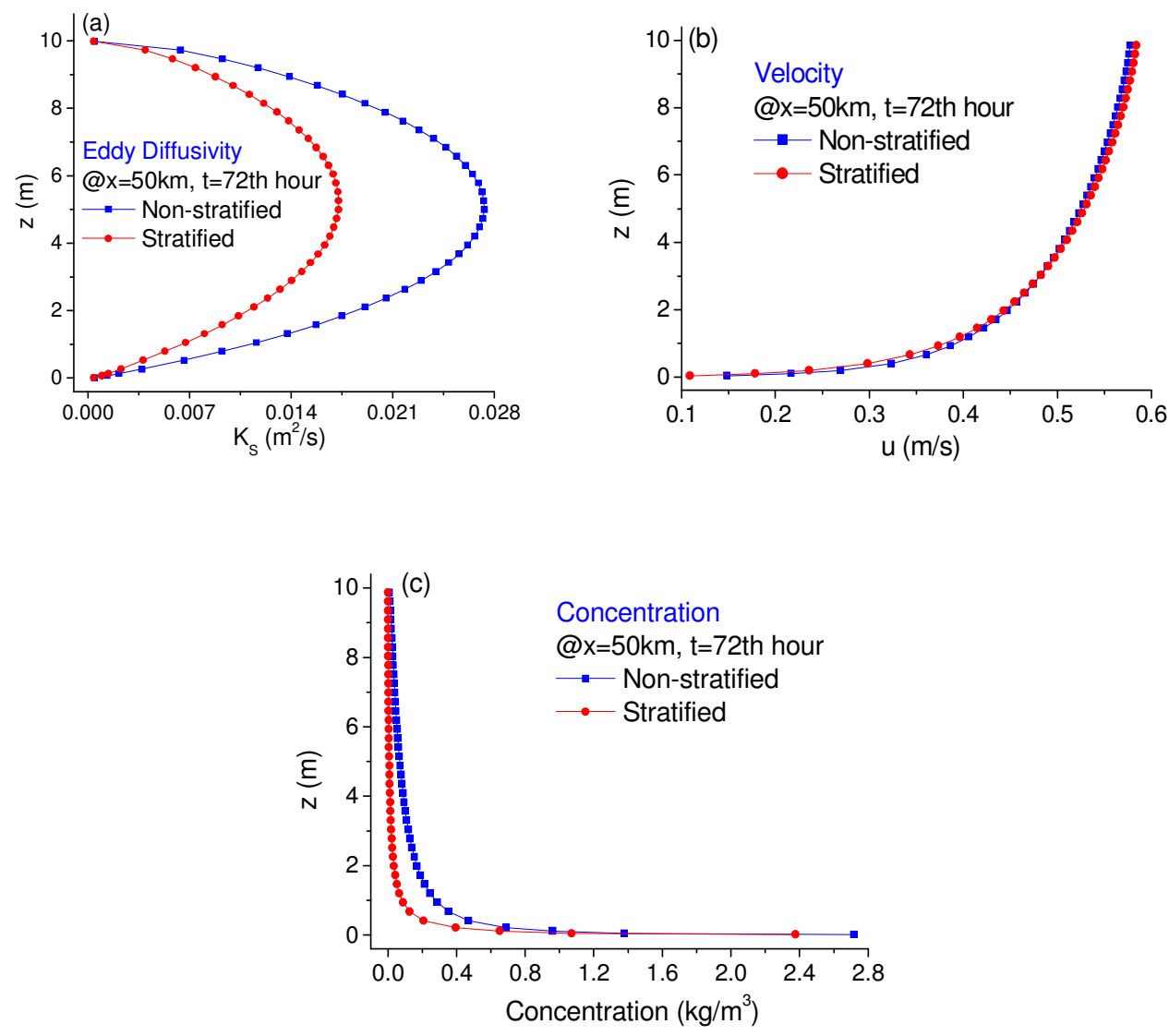

Figure 4 Predicted vertical profiles of (a) eddy viscosity; (b) Velocity; (c) Concentration above the wave boundary layer at the center of domain. Sediment size is $0.1 \mathrm{~mm}$. 
Due to the reduced current shear velocity, the velocity gradient near the bottom decreases, but this tendency is counteracted by the reduced mixing due to stable stratification which would tend to increase the velocity gradient. The combined effect of these processes is a decrease of velocity near the bottom and, since the depth-averaged velocity has to be maintained at $0.5 \mathrm{~m} / \mathrm{s}$, a slight increase in the upper layers (seen in Figure 4b). For example, calculations suggest that the velocity for stratified case is reduced by $27 \%$ (from $14.8 \mathrm{~cm} / \mathrm{s}$ to $10.9 \mathrm{~cm} / \mathrm{s}$ ) at bottom to about $1 \%$ at $2 \mathrm{~m}$ above the bed and the difference is less than about $1.2 \%$ in the rest portion of the water column.

Since a reduction in shear velocity leads to a smaller reference concentration and stable stratification reduces upward mixing of sediments into the water column, the two effects mentioned above both result in a decrease in suspended sediment concentration. One can therefore expect a significant reduction in the sediment concentration and transport rates due to self-stratification effects. The reference concentrations in Table 1 show that these are reduced by about $7 \%$ from $7.23 \mathrm{~kg} / \mathrm{m}^{3}$ to $6.75 \mathrm{~kg} / \mathrm{m}^{3}$ at $z=7 d$. This very minor reduction is, of course, associated with the virtually unchanged wave shear velocities, which dominate in the creation of the mean reference concentration. Consequently, the concentration within the boundary layer does not show significant reduction as seen in Figure 3b. In contrast, the concentration above the wave boundary layer (shown in Figure 4c) is significantly decreased by the stratification effect, e.g. the concentration at $1 \mathrm{~m}$ above the bed is reduced by about $68 \%$ from $0.28 \mathrm{~kg} / \mathrm{m}^{3}$ to $0.09 \mathrm{~kg} / \mathrm{m}^{3}$, although the difference between non-stratified and stratified cases is only $13 \%$ at bottom.

The transport rates in Table 2 reveal that the net bed-load and mean suspended load transport rates within the boundary layer decrease only by roughly $30 \%$. The relatively small reduction of net bed-load transport rate is because it depends only on the sediment transport shear stress which, as seen from Table 1, does not change appreciably. The reason for the slight reduction of mean suspended load transport rate within the boundary layer reflects primarily the reduction in current velocity (shown in Figure 3a), whereas the concentration (Figure 3b), predicted without considering stratification, hardly changes. However, the suspended load transport rate above the boundary layer is significantly reduced as a result of stratification. The stratified value of $0.12 \mathrm{~kg} / \mathrm{m} / \mathrm{s}$ is only $24 \%$ of the $0.5 \mathrm{~kg} / \mathrm{m} / \mathrm{s} \mathrm{predicted}$ when stratification effects are neglected. This significant influence of stratification effects above the boundary layer is mainly caused by the reduction of turbulent mixing associated with the stable stratification and, to a lesser extent, by the reduced current shear stress.

\subsection{Effects of Sediment Diameter}

In the preceding sections, we discussed wind wave and stratification effects based on numerical results for $0.1 \mathrm{~mm}$ sediment. To investigate the influence of sediment size on sediment transport, we perform two simulations, one with and the other without stratification effects, for sediment of $0.2 \mathrm{~mm}$ diameter. The same flow and wave conditions as those for the $0.1 \mathrm{~mm}$ sediment are used, and results are listed in Tables 1 and 2.

As shown in Table 1, the physical and apparent roughness are about $12 \mathrm{~cm}$ and $52 \mathrm{~cm}$. These are significantly larger than those for the $0.1 \mathrm{~mm}$ sediment, indicating larger ripples and therefore smaller ratios between skin friction shear velocities and total shear velocities. This is confirmed by the results in Table 1, e.g. the ratio of skin friction and total current shear stress for non-stratified case is 0.26 for $0.2 \mathrm{~mm}$ sediment compared to 0.46 for $0.1 \mathrm{~mm}$ sediment. The reference concentrations at $z=7 d$ are quite similar for the $0.1 \mathrm{~mm}$ and $0.2 \mathrm{~mm}$ sediments; however, due to its much larger fall velocity, the mean concentration at the outer edge of the bottom boundary layer, $0.75 \mathrm{~kg} / \mathrm{m}^{3}$ for $0.2 \mathrm{~mm}$ sediment, is only $28 \%$ of the value for $0.1 \mathrm{~mm}$ sediment.

Due to the near-identical critical shear stresses and much different fall velocities for the two sediments, the difference of net bed-load transport rates between the two sediments is smaller than that of suspended load transport rates. As shown in Table 2, the difference of net bed-load transport rates for non-stratified case is about $38 \%$, whereas the difference between suspended transport rates above the boundary layer is as large as $97 \%$.

As for the stratification effect, the results in Table 2 show that the stratification only results in a $12 \%$ reduction of total sediment transport rate for $0.2 \mathrm{~mm}$ sediment, i.e. much less than the $75 \%$ reduction found for the $0.1 \mathrm{~mm}$ sediment. The primary reason for this is that the concentration above the boundary layer is significantly reduced for the $0.2 \mathrm{~mm}$ sediment due to its much larger fall velocity, $2.2 \mathrm{~cm} / \mathrm{s}$ vs. $0.72 \mathrm{~cm} / \mathrm{s}$, and therefore generates less stratification effect. 


\section{CONCLUSIONS}

In this paper, a three dimensional sediment transport model is proposed for the computation of sediment transport in combined wave-current flows in coastal waters. The model calculates three contributions to the total sediment transport rate: (i) net bed-load transport in the bed-load layer $z<7 d$; (ii) mean suspended load transport within the bottom boundary layer $7 d<z<\delta_{c w}$; and (iii) suspended load transport above the wave boundary layer $z>\delta_{c w}$. Wind wave effects on flow and sediment transport are accounted for through an analytical bottom boundary layer model. For the hydrodynamics, the presence of wind waves enhances the flow resistance by increasing the roughness, the so-called apparent roughness, experienced by a current in the presence of waves. For the sediment transport processes, wind waves increase the bottom shear stress and the near-bed turbulence and therefore mobilize and suspend more sediment than would be the case if the waves were absent. In addition, the wave enhanced turbulent mixing in the water column above the wave boundary layer diffuse more sediment up into the water column. In the water column above the wave boundary layer hydrodynamics and sediment transport processes are solved numerically using POM and stratification effects induced by suspended sediment are taken into account. Idealized tests were performed to examine the model's ability to predict sediment transport in combined wave-current flows.

The test results reveal that wind waves have a pronounced effect on hydrodynamics and suspended sediment transport for typical conditions of coastal waters, e.g. sediment may only be mobilized and transported when wind waves are present. The sediment transport rate within the wave boundary layer, which has been neglected in most previous studies, is more important for coarser sediment, e.g. it is about $20 \%$ the suspended load transport above the boundary layer for $0.2 \mathrm{~mm}$ sediment compared to $1 \%$ for $0.1 \mathrm{~mm}$ sediment case in the present study's computational example. Therefore, wind wave effects should never to be neglected in sediment transport modeling in coastal waters.

The numerical tests suggest that the self-stratification effect is more significant for fine sediment as it causes $75 \%$ drop in total transport rate for the $0.1 \mathrm{~mm}$ sediment case, but becomes less significant for coarser sediment as the reduction of total transport rate is only $12 \%$ for $0.2 \mathrm{~mm}$ sediment. Essentially, the stratification effect can be very significant when concentration gradients are large, which usually happens for relatively fine sediment and strong flow conditions. In such circumstances, our results show that the self-stratification effect should be accounted for when modeling sediment transport in coastal waters.

It is noted that the present model tests were performed for a steady co-directional wave-current flow. Further tests must be conducted to test the model's capabilities in more realistic flows, e.g. in unsteady flows and in the cases where waves and current are in different directions. For unsteady, slowly varying flows, the applicability of the concentration bottom boundary condition, which assumes equilibrium conditions, needs to be tested. To more accurately predict the sediment transport in combined wave-current flows, the wave-associated suspended load transport within the wave boundary

layer, $\tilde{q}_{S 1}=\int_{7 d}^{\delta_{c w}} \tilde{u} \tilde{C} d z$, with $\tilde{u}(t)$ and $\tilde{C}(t)$ denoting the time-varying wave-associated velocity and concentration within the wave boundary layer, should be included. Considering the significant influence of self-stratification on suspended transport above the boundary layer, it would be prudent to examine the effect of stratification within the wave boundary layer, or in the extrapolation of the mean concentration from the outer edge of the wave boundary layer to the numerical model's grid point where a reference concentration is specified. We are currently pursuing extensions of the sediment transport model along these lines as well as examining its performance in more realistic scenarios.

\section{ACKNOWLEDGMENTS}

The research described in this paper was funded in whole or in part by the Singapore National Research Foundation (NRF) through the Singapore-MIT Alliance for Research and Technology's (SMART) Center for Environmental Sensing and Modeling (CENSAM).

\section{REFERENCES}

Blumberg, A.F., B. Galperin, and D.J. O'Connor. 1992. Modeling vertical structure of open-channel flows, Journal of Hydraulic Engineering, 118(H8), 1119-1134.

Blumberg, A.F., and G.L. Mellor. 1987. A description of a three-dimensional coastal ocean circulation model, Three Dimensional Coastal Ocean Models, N. Heaps, Ed., Coastal Estuarine Science, Vol. 4, Amer. Geophys. Union, 1-16. 
Grant, W.D., and O.S. Madsen. 1979. Combined wave and current interaction with a rough bottom, Journal of Geophysical Research, 84(C4), 1797-1808.

Herrmann, M.J., and O.S. Madsen. 2007. Effect of stratification due to suspended sand on velocity and concentration distribution in unidirectional flows, Journal of Geophysical Research, 112, C02006, doi:10. 1029/2006JC003569.

Humbyrd, C.J., and O.S. Madsen. 2010. Predicting movable bed roughness in coastal waters, Proceedings of the International Conference on Coastal Engineering. No. 32(2010), Shanghai, China. Paper \#: sediment.6. Retrievable from http://journals.tdl.org/ICCE/

Jiménez, J.A. and O.S. Madsen. 2003. A simple formula to estimate settling velocity of natural sediment, Journal of waterway, port, coastal and ocean engineering, 129, No.2, 70-78.

Lesser, G.R., J.A. Roelvink, J.A.T.M. van Kester, and G.S. Stelling. 2004. Development and validation of a three-dimensional morphological model, Coastal Engineering, 51, 883-915.

Li, M.Z. and C.L. Amos. 2001. SEDTRANS96: the upgraded and better calibrated sediment-transport model for continental shelves, Computers \& Geosciences, 27(6), 619-645.

Madsen, O.S. (1991) Mechanics of cohesionless sediment transport in coastal waters. Proceedings of Coastal Sediments '91, ASCE, Seattle, USA. 1:15-27

Madsen, O.S. 1994. Spectral wave-current bottom boundary layer flows. Proceedings of $24^{\text {th }}$ International Conference on Coastal Engineering, ASCE, 384-398.

Madsen, O.S. 2002. Sediment Transport Outside the Surf Zone. In: Walton, T. (editor), Coastal Engineering Manual, Part III, Coastal Processes, Chapter III-6, Engineer Manual 1110-2-1100, U.S. Army Corps of Engineers, Washington, DC.

Mellor, G.L., and T. Yamada. 1982. Development of a turbulence closure model for geophysical fluid problems, Review of Geophysics and Space Physics, 20, 851-875.

Warner, J.C., C.R. Sherwood, H.G. Arango, and R.P. Signell. 2005. Performance of four turbulence closure models implemented using a generic length scale method, Ocean Modelling, 8, 81-113.

Warner, J.C., C.R. Sherwood, R.P. Signell, C.K. Harris, and H.G. Arango. 2008. Development of a three-dimensional, regional, coupled wave, current and sediment-transport model, Computers \& Geosciences, 34, 1284-1306. 